\title{
Mechanisms Involved in Aggravation of Ethanol-Induced Gastric Mucosal Lesions in Adrenalectomized Rats
}

\author{
Hideyuki NISHIWAKI, Megumu OKADA, Nobuyuki HARA, \\ Koji TAKEUCHI* and Susumu OKABE \\ Department of Applied Pharmacology, Kyoto Pharmaceutical University, \\ Misasagi, Yamashina, Kyoto 607. Japan
}

Accepted September 19, 1988

\begin{abstract}
Effects of adrenalectomy (AD) on ethanol-induced gastric injury and prostaglandin (PG) protection on the damage were investigated in rats and compared with those of $\mathrm{N}$-ethylmaleimide (NEM), a sulfhydryl (SH) blocker, and diethyl maleate (DEM), a SH depletor. Oral administration of $100 \%$ ethanol $(1 \mathrm{ml})$ induced elongated bands of hemorrhagic lesions in the corpus mucosa of sham operated rats, and these lesions were significantly prevented by 16,16-dimethyl $\mathrm{PGE}_{2}$ (dmPGE, $10 \mu \mathrm{g} / \mathrm{kg}$, s.c.). AD markedly enhanced the mucosal ulcerogenic responses caused by ethano! and abolished the protective effect of $\mathrm{dmPGE}_{2}$; this agent rather worsened the lesions, which appeared throughout the corpus mucosa. $A D$ by itself enhanced the microvascular permeability in the gastric mucosa without any effect on SH contents. These alterations caused by AD were significantly reverted by hydrocortisone treatment $(10 \mathrm{mg} / \mathrm{kg} / \mathrm{day}$ for 2 weeks, s.c.). On the other hand, a single injection of NEM $(10 \mathrm{mg} / \mathrm{kg}$, s.c.) similarly enhanced the vascular permeability, worsened the ethanol-induced lesion, and mitigated the protective effect of $d m \mathrm{PGE}_{2}$ without altering mucosal SH contents, while DEM (1 $\mathrm{ml} / \mathrm{kg}$, s.c.) significantly reduced the mucosal $\mathrm{SH}$ levels and the lesions. These results suggest that $A D$ worsened the mucosal lesions induced by ethanol, probably by enhancing the microvascular permeability, and this action may be due to a lack of steroid secretion but is not directly related to a mucosal $\mathrm{SH}$ deficiency.
\end{abstract}

Recent studies have shown that the adrenal glands modified the pathogenesis of gastric mucosal lesions in a variety of experimental ulcer models. Hernandez et al. (1) showed that adrenalectomy reduced the protective action of neurotensin against stress-induced gastric lesions in rats. Szabo et al. (2) also reported a significant attenuation of the mucosal protective action of prostaglandin $(P G) F_{2 \beta}$ against gastric damage induced by ethanol. On the other hand, glucocorticoids protected the mucosa from ethanol insult (3) and restored the mucosal protective effect of $P G_{2 \beta}(2)$. However, the mechanism for how adrenalectomy produced such alterations in the mucosal ulcerogenic responses remains unknown.

\footnotetext{
* To whom correspondence should be addressed.
}

Szabo et al. (4) and Pihan et al. (5) demonstrated a significant prevention by 16,16dimethyl $P G E_{2} \quad\left(d m P G E_{2}\right.$ ) and a sulfhydryl $(\mathrm{SH})$ drug against the increased vascular permeability and gastric lesions caused by ethanol; and they proposed that endogenous $\mathrm{SH}$ s and vascular endothelium may be considered as a protector and a target, respectively, in the pathogenesis of acute gastric mucosal injury.

The present study was therefore designed to investigate factors responsible for pathogenetic modification caused by adrenalectomy in rats using ethano!-induced gastric lesions and to compare the effects of adrenalectomy with those of $\mathrm{N}$-ethylmaleimide (NEM), a SH blocker (6). We discussed the influences of adrenalectomy on the basis of mucosal vascular permeability and endo- 
genous SH contents.

\section{Materials and Methods}

Male Sprague Dawley rats (200-230 g) were used in all experiments. Each study was carried out using 6 to 16 rats per group.

General protocols: The experiments were performed in the following three groups: A. sham operation, B. adrenalectomy, C. adrenalectomy plus hydrocortisone treatment. In groups $B$ and $C$, bilateral adrenalectomy was performed by a translumber route under ether anesthesia, and the adrenal glands were removed carefully by forceps. The animais were fed on normal chow and $2 \% \mathrm{NaCl}$ solution after the operation. In group $\mathrm{C}$, the animals received hydrocortisone acetate subcutaneously once daily for 2 weeks (Sigma Chemicals, $10 \mathrm{mg} / \mathrm{kg} /$ day) after the operation (2). In group A, bilateral body walls at the lumber portion were incised, the incisions were closed without removing the adrenal glands, and the animals were fed on normal chow and tap water. The animals in each group were used in the experiments 2 weeks after the operation, and they were deprived of food for $18 \mathrm{hr}$ prior to the experiments. In these three groups, the mucosal ulcerogenic response to ethanol, mucosal permeability, and mucosal sulfhydryl (SH) contents were measured. In a parallel study, the effects of $\mathrm{N}$-ethylmaleimide (NEM), a SH blocker, were examined on the above parameters and compared with those of adrenalectomy.

Induction of gastric mucosal injury by ethanol: The animals were given $1 \mathrm{ml}$ of absolute ethanol orally by esophageal intubation, and they were killed 1 hr later. The stomachs were removed, inflated by injecting $10 \mathrm{ml}$ of $2 \%$ formalin, immersed in $2 \%$ formalin for $10 \mathrm{~min}$ to fix the gastric wall, and opened along the greater curvature. The area $\left(\mathrm{mm}^{2}\right)$ of the reddish part of the mucosa was measured under a dissecting microscope with a square grid $(\times 10)$, and the lesion index was expressed as the percentage (\%) of such areas to the total glandular mucosal area. 16. 16-Dimethyl prostaglandin $E_{2} \quad\left(d m P G E_{2}\right.$ : Funakoshi, $10 \mu \mathrm{g} / \mathrm{kg}$ ) was given subcutaneously $30 \mathrm{~min}$ before administration of ethanol. NEM (Sigma, $10 \mathrm{mg} / \mathrm{kg}$ ) or diethyl maleate (DEM; Sigma, $1 \mathrm{ml} / \mathrm{kg}$ ), a $\mathrm{SH}$ depletor, was given subcutaneously $1 \mathrm{hr}$ before ethanol treatment. In each study, the person measuring the lesions did not know the treatment given to the animals.

Measurement of mucosal vascular permeability: The mucosal vascular permeability was determined using a dye according to a previous study (7). In the animals subjected to sham operation, adrenalectomy, adrenalectomy plus hydrocortisone, and treatment with NEM, DEM or dmPGE, $1 \mathrm{ml}$ of Evans blue (Sigma, $1 \% \mathrm{~W} / \mathrm{W}$ ) was injected intravenously, and they were killed $30 \mathrm{~min}$ later. NEM, DEM or dmPGE 2 was given subcutaneously $1 \mathrm{hr}$ before sacrifice of the animals. Under ether anesthesia, the animals were killed by bleeding from the descending aorta, the stomachs were removed, and the amount of dye trapped for $30 \mathrm{~min}$ in the corpus mucosa and in the gastric contents was measured. After collecting the gastric contents carefully by lavaging with $5 \mathrm{ml}$ of distilled water, the stomach was opened along the greater curvature and the corpus mucosa was scraped off using two glass slides, weighed and put into a tube containing $5 \mathrm{ml}$ of distilled water. The extraction of dye was performed according to the method described by Katayama et al. (8), and the absorbance of samples was measured at $620 \mathrm{~nm}$ in a Hitachi spectrophotometer (Model 200-100). The amount of dye recovered from the gastric contents and the corpus mucosa was expressed as micrograms per milliliter of gastric juice and micrograms per $100 \mathrm{mg}$ of tissue, respectively.

Measurement of mucosal sulfhydryl contents: The amount of non-protein $\mathrm{SH}$ was measured in the gastric mucosa of rats following adrenalectomy with or without hydrocortisone treatment, and administration of NEM, DEM or dmPGE $E_{2}$, according to the modified method described by Kaplowitz et al. (9). The animals were killed 2 weeks after adrenalectomy or $2 \mathrm{hr}$ after treatment with the above drugs. The stomachs were removed and incised along the greater curvature, and the corpus mucosa was scraped off using two glass slides and kept cold on ice. The mucosal scrapings were weighed, homogenized in 2 $\mathrm{ml}$ of phosphate buffer $\left(0.1 \mathrm{~N} \mathrm{NaPO}_{4}\right.$ plus $0.25 \mathrm{~N}$ sucrose, $\mathrm{pH}$ 7.4), and centrifuged at 
4000 r.p.m. for $15 \mathrm{~min}$ at $4^{\circ} \mathrm{C}$. Then, $0.5 \mathrm{ml}$ of $25 \%$ trichloroacetic acid was added to $1 \mathrm{ml}$ of the supernatant of each sample and allowed to stand for $30 \mathrm{~min}$. After centrifugation for $15 \mathrm{~min}$ at 3000 r.p.m., the supernatant was used to determine SH using DNTB $\left(5-5^{\prime}\right.$ dithio-2-nitrobenzoic acid, Wako). Absorbance was measured at $412 \mathrm{~nm}$ in a Hitachi spectrophotometer, and the results were expressed as micromoles per $g$ wet tissue weight.

Preparation of drugs: Both NEM and DEM were dissolved in saline, while dmPGE 2 was first dissolved in absolute ethanol and diluted

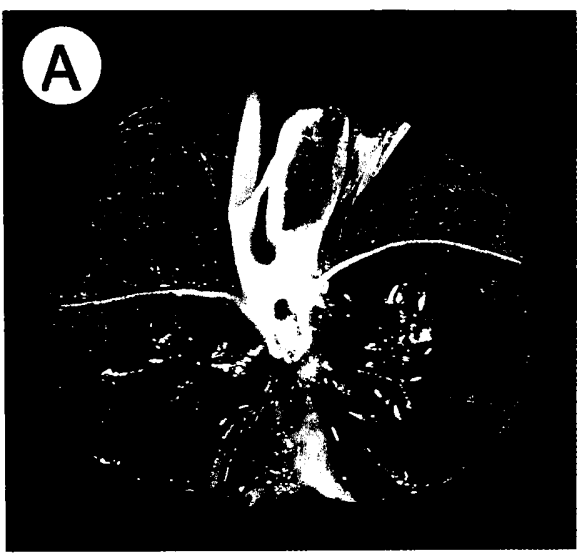

Sham Operated Rat with saline to the desired concentration. Hydrocortisone acetate was suspended in saline with a trace of Tween 80 (Nakarai). Each drug was prepared immediately before use and given in a volume of $0.5 \mathrm{ml}$ per $100 \mathrm{~g}$ of body weight. Control animals received saline in the same volume.

Statistics: Data are presented as the mean \pm S.E. from 6 to 16 rats per group. Statistical analysis was performed using a two tailed Dunnett's multiple comparison test (10), and values of $P<0.05$ were regarded as significant.

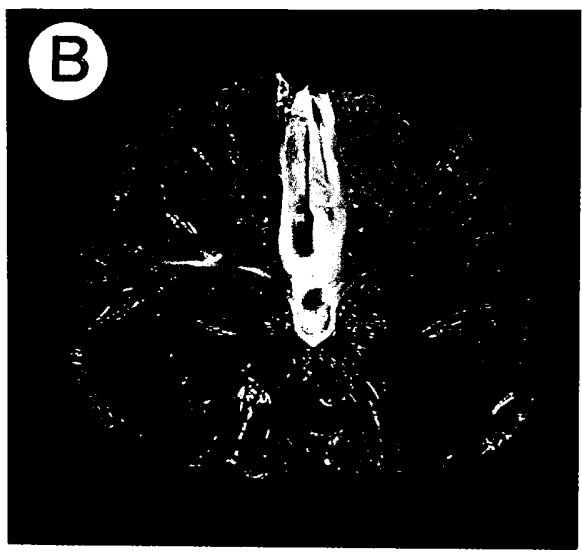

Adrenalectomized Rat

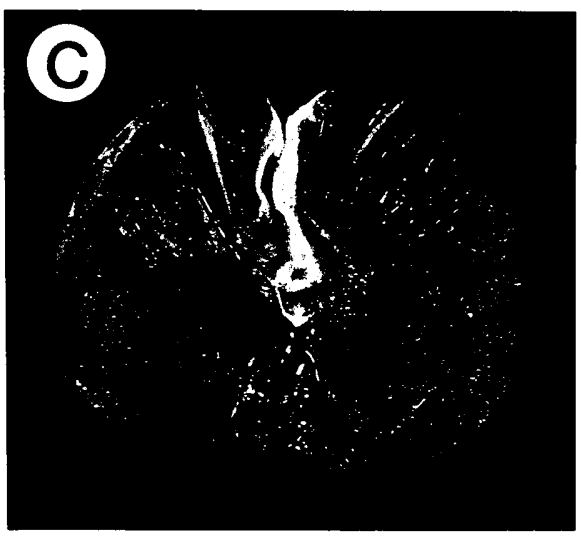

$\mathrm{dmPGE}, 10 \mu \mathrm{g} / \mathrm{kg}$

Fig. 1. Gross appearance of gastric mucosal lesions induced by ethanol in rats. Animals were given $1 \mathrm{ml}$ of $100 \%$ ethanol orally by esophageal intubation, and they were killed $1 \mathrm{hr}$ later. DMPGE 2 (10 $\mu \mathrm{g} /$ $\mathrm{kg}$ ) was given subcutaneously $30 \mathrm{~min}$ before ethanol treatment. Figures show: A, sham operated rat: $B$, adrenalectomized rat; $C, d m P G E_{2}$-treated adrenalectomized rat. Note that adrenalectomy not only worsened ethanol-induced lesions but also abolished the protective effect of $\mathrm{dmPGE} \mathrm{m}_{2}$ on these lesions. 


\section{Results}

Influences of adrenalectomy on ethanolinduced gastric mucosal lesions: Oral administration of absolute ethanol in sham operated rats caused multiple elongated bands of hemorrhagic lesions in the corpus mucosa within $1 \mathrm{hr}$ (Fig. 1A), the lesion index being $10.8 \pm 2.1 \%$ of the mucosa. Gastric lesions induced by ethanol were markedly worsened in the animals subjected to bilateral adrenalectomy, and the lesion index reached about 3 times greater than the control (Figs. 1 and 2A). Of interest, while subcutaneously administered dmPGE $2(10 \mu \mathrm{g} / \mathrm{kg})$ almost completely prevented the formation of gastric lesions in response to ethanol in sham operated rats, this agent failed to afford mucosal protection in adrenalectomized rats and rather worsened these lesions. In these animals treated with dmPGE 2 , the injury was not localized as band-like lesions but was ob-
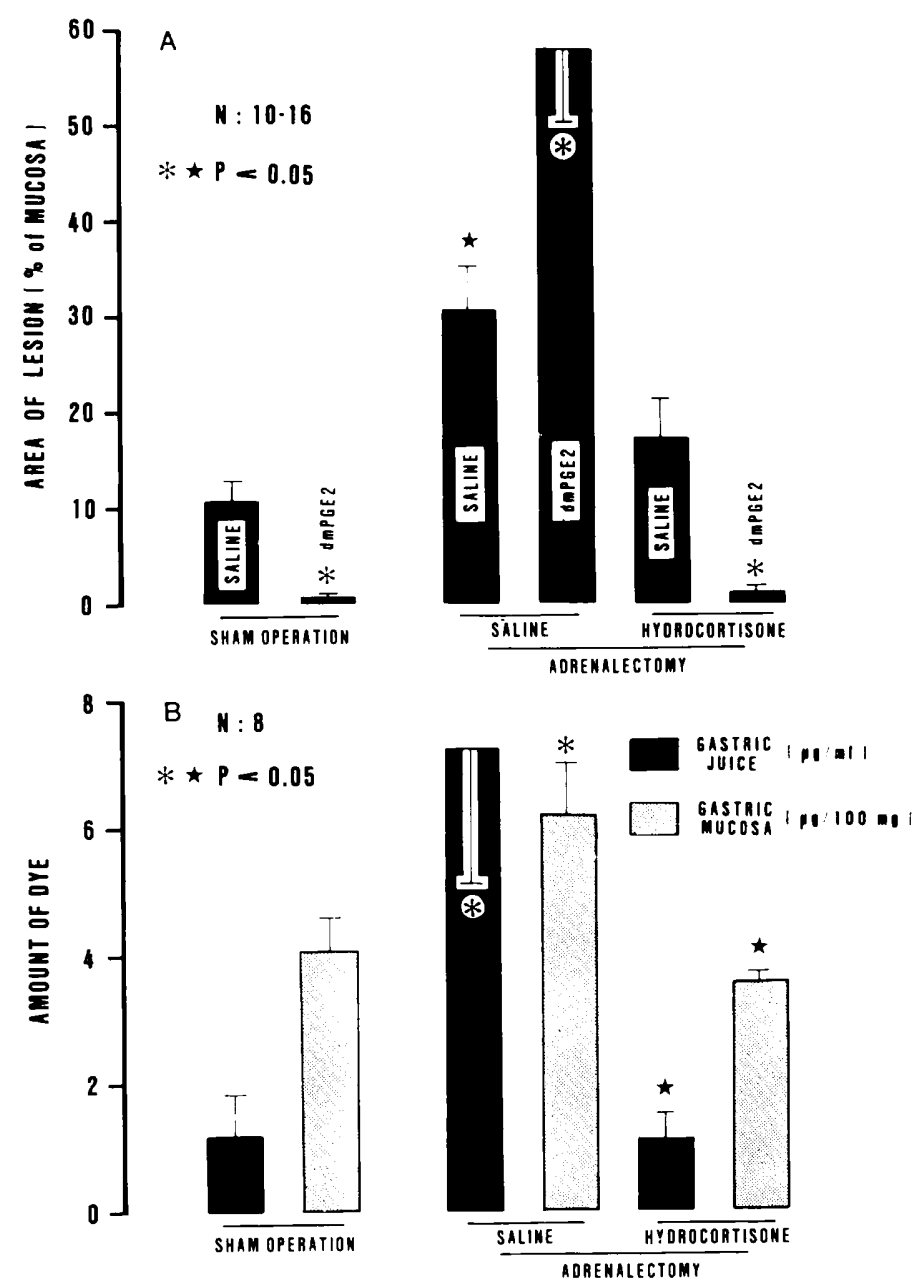

Fig. 2. Effects of adrenalectomy on ethanol-induced gastric lesions and the protective effect of dmPGE against these lesions (A) and on the mucosal vascular permeability (B) in rats. Hydrocortisone (10 mg/ $\mathrm{kg} /$ day) was given subcutaneously once daily for 14 days after adrenalectomy. In Fig. A, ethanol (1 ml, $100 \%)$ was given orally by esophageal intubation, and $\mathrm{dmPGE}_{2}(10 \mu \mathrm{g} / \mathrm{kg})$ was given subcutaneously $30 \mathrm{~min}$ before ethanol treatment. Data are presented as the mean \pm S.E. from 8 to 16 rats per group. * $\star$ Statistically significant difference from the corresponding controls, at $\mathrm{P}<0.05$. 
served as more generalized lesions (see Fig. 1C). The worsening effect of adrenalectomy on ethanol-induced gastric lesions was almost totally attenuated by treatment of these animals with hydrocortisone $(10 \mathrm{mg} / \mathrm{kg} /$ day for 2 weeks), and in these animals, dmPGE 2 again showed a potent mucosal protection against ethanol.

Effects of sulfhydryl blocker and depletor on ethanol-induced gastric mucosal lesions: In normal rats, gastric lesions induced by ethanol were significantly inhibited by both $\mathrm{dmPGE}_{2}(10 \mu \mathrm{g} / \mathrm{kg})$ and $\operatorname{DEM}(1 \mathrm{ml} / \mathrm{kg})$, but markedly aggravated by pretreatment with NEM (10 mg/kg) (Fig. 3A). Gastric lesions induced by ethanol in the NEM-treated animals appeared in wide bands of lesions along the long axis of the stomach, similar to those observed in adrenalectomized rats. Of interest, pretreatment of the animals with NEM completely abolished the protective activity of $\mathrm{dmPGE}$, and the lesions rather significantly worsend as compared to the control group.

Effects of adrenalectomy and NEM on gastric mucosal vascular permeability: The
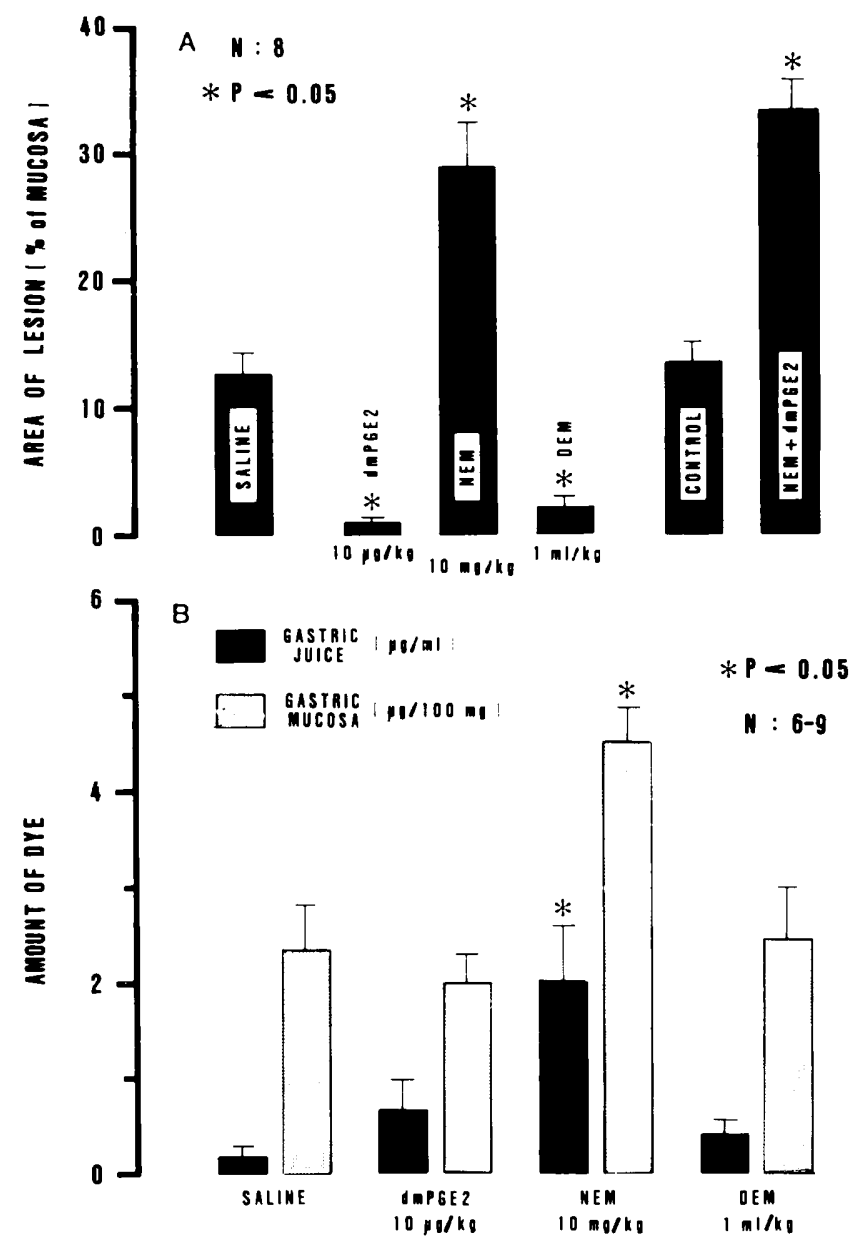

Fig. 3. Effects of dmPGE $(10 \mu \mathrm{g} / \mathrm{kg})$, NEM $(10 \mathrm{mg} / \mathrm{kg})$ and DEM $(1 \mathrm{ml} / \mathrm{kg})$ on ethanol-induced gastric mucosal lesions (A) and on the vascular permeability (B) in rats. In Fig. A, ethanol $(1 \mathrm{ml}, 100 \%)$ was given orally by esophageal intubation; and NEM. DEM or $\mathrm{dmPGE}_{2}$ was given subcutaneously $1 \mathrm{hr}$ or $30 \mathrm{~min}$, respectively, before ethanol treatment. In the combined treatment of dmPGE 2 plus NEM, the former was given $30 \mathrm{~min}$ before administration of NEM. Data are presented as the mean $\pm S$.E. from 6 to 9 rats per group. *Statistically significant difference from the controls, at $\mathrm{P}<0.05$. 

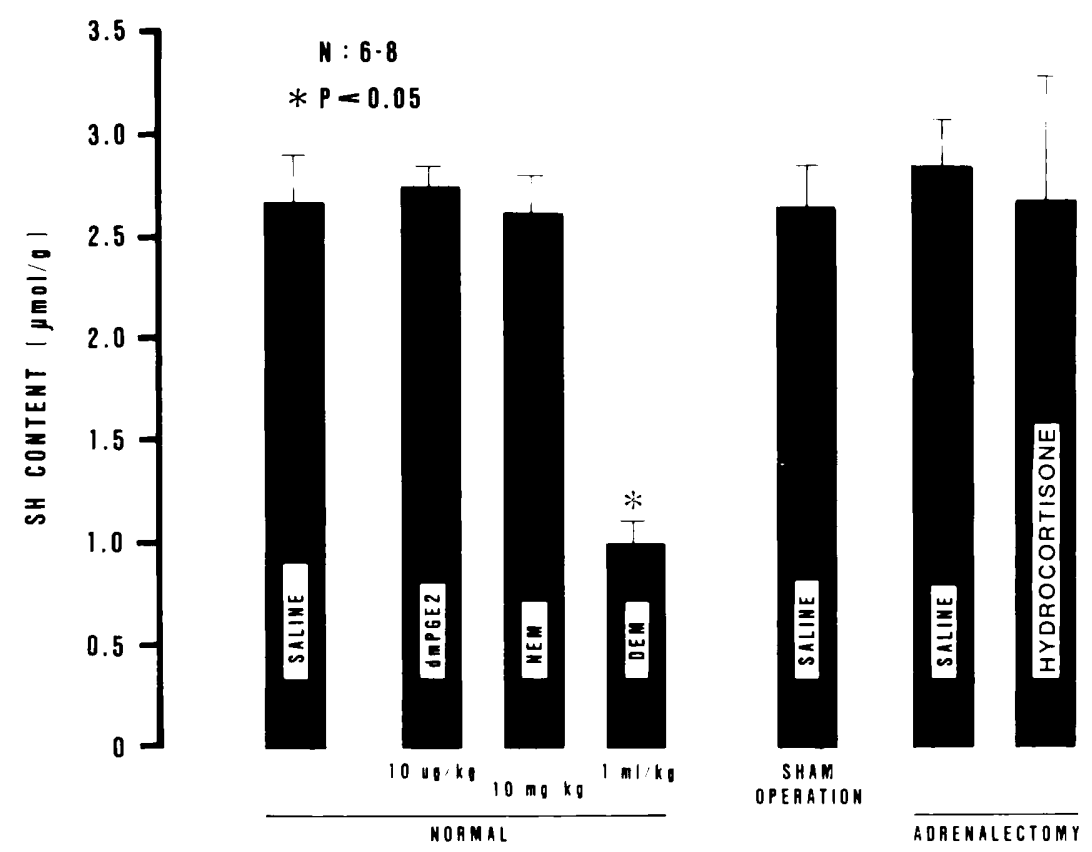

Fig. 4. Effects of adrenalectomy and various agents on gastric mucosal sulfhydryl (SH) levels in rats. $\mathrm{dmPGE}(10 \mu \mathrm{g} / \mathrm{kg})$, NEM $(10 \mathrm{mg} / \mathrm{kg})$ or DEM $(1 \mathrm{ml} / \mathrm{kg})$ was given subcutaneously, and hydrocortisone $(10 \mathrm{mg} / \mathrm{kg} /$ day) was given subcutaneously once daily for 14 days after adrenalectomy. The animals were killed $2 \mathrm{hr}$ after administration of each drug. Data are presented as the mean \pm S.E. from 6 to 8 rats per group. *Statistically significant difference from the controls, at $P<0.05$.

vascular permeability of the gastric mucosa under normal conditions was minimal when determined as an accumulation of Evans blue in both the gastric mucosa and contents, the values being $0.5-1.7 \mu \mathrm{g} / \mathrm{ml}$ of gastric juice and 3-4 $\mu \mathrm{g} / 100 \mathrm{mg}$ tissue (Figs. 2B and $3 \mathrm{~B}$ ). Bilateral adrenalectomy significantly increased the mucosal vascular permeability, and the amount of dye in the gastric contents was increased to about 5 -fold greater than the control values. The increased vascular permeability caused by adrenalectomy was significantly suppressed by treatment with hydrocortisone, and the values were not statistically significant from those observed in normal rats. Neither dmPGE 2 nor DEM had any effect on the mucosal vascular permeability, but NEM did significantly augment the amount of dye accumulated in both gastric mucosa and contents.

Effect of adrenalectomy and NEM on mucosal SH contents: The levels of mucosal $\mathrm{SH}$ (non-protein sulfhydryls) in normal rat stomachs were $2.54 \pm 0.32 \mu \mathrm{mol} / \mathrm{g}$ tissue, and they remained in the same range following sham operation or bilateral adrenalectomy (Fig. 4). Treatment of the animals with either dmPGE 2 . NEM or hydrocortisone did not significantly affect the $\mathrm{SH}$ contents in the gastric mucosa, while administration of DEM significantly reduced the levels of mucosal $\mathrm{SH}$ to $1 / 3$ of the normal values.

\section{Discussion}

The present study confirmed the finding by Szabo et al. (2), who showed that adrenalectomy in rats inhibits the usual protective effect of a PG on ethanol-induced injury of the gastric mucosa, and the results further suggested a possible involvement of the increased vascular permeability caused by adrenalectomy in this phenomenon.

A relationship between the adrenal glands and gastric lesions has been postulated in experimental animals using stress ulcers, indomethacin-induced lesions and ethanolinduced gastric lesions $(1,2,11)$. Most of the studies showed aggravation of the lesions 
following adrenalectomy, although the mechanism remains unknown. Szabo et al. (2) demonstrated that adrenalectomy attenuated the usual protective effect of $\mathrm{PGF}_{2 \beta}$ and $\mathrm{SH}$ drugs or cimetidine on ethanol- or aspirininduced gastric lesions. In agreement with those data, the present study showed that adrenalectomy by itself worsened ethanolinduced gastric lesions but also attenuated the protective effect of $d m P G E_{2}$ against these lesions. These findings suggest that adrenalectomy produces a generalized worsening effect, irrespective of the types of lesions or the types of antiulcer drugs. Since the medullectomy had no effect on indomethacin-induced gastric lesions (11) and rather inhibited ethanol-induced gastric injury (2), it seems likely that the worsening effect of adrenalectomy is due to removal of the adrenal cortex. This notion is supprted by the present finding that replacement with hydrocortisone significantly prevented the pathogenetic modification of ethanol injury in the adrenalectomized animals.

Ethanol-induced gastric lesions appeared usually as elongated bands of damage along the long axis of the stomach. Adrenalectomy induced more wide bands of the lesions in response to ethanol, and it caused more generalized lesions throughout the corpus mucosa in the presence of dmPGE 2 . Recent studies indicate that microvascular alterations may play a critical role in acute gastric mucosal damages. Intragastric administration of ethanol also results in rapidly developing vascular injury in subepithelial capillaries and increases the vascular permeability as measured by Evans blue extravasation $(4,12)$. Of interest, adrenalectomy significantly increased the amount of extravasated dye in the mucosa, suggesting a possible involvement of enhanced vascular permeability in the mechanism through which ethanol-induced lesions worsened. Since dmPGE 2 alone slightly increased the vascular permeability and is know to potentiate the permeability responses caused by other agents $(6,13)$, the permeability alterations may be associated with the pathogenetic modification observed in the adrenalectomized rats. This contention is supported by the findings that NEM also increased the vascular permeability, worsened the ethanol-induced lesions, and attenuated the protective effect of $d \mathrm{PPGE}_{2}$. In addition, glucocorticoid treatment significantly reversed the enhanced vascular permeability and mucosal ulcerogenic responses caused by adrenalectomy. Since glucocorticoids are known to induce endogenous factors (vasoregulin) which inhibit the vascular permeability (14), a lack of steroid secretion induces the vascular fragility, the increased vascular permeability, and thereby enhances the mucosal ulcerogenic response caused by ethanol.

On the other hand, it has been suggested that mucosal $\mathrm{SH}$ is considered to be a protector against gastric damage induced by necrotizing agents (15). Thus, it is possible to assume that adrenalectomy modified ethanolinduced mucosal lesions by means of reducing endogenous $\mathrm{SH}$ levels. However, the present study excludes the above possibility for the following reasons: (a) both adrenalectomy and NEM significantly worsened ethanol-induced gastric lesions without altering the mucosal SH levels. (b) DEM, a SH depletor, significantly reduced $\mathrm{SH}$ contents in the gastric mucosa, yet inhibited the mucosal lesions induced by ethanol. We found that the functional pertubations of endogenous SH by NEM markedly enhanced the microvascular permeability responses and mucosal lesions induced by ethanol. Especially, this agent significantly augmented the microvascular permeability in normal rats without exposure to ethanol, suggesting that alkylation of $\mathrm{SH}$ makes the vasculature fragile to adversely affect the permeability of endothelial or parenchymal cells. Since recent studies showed that $\mathrm{SH}$ are involved in nonreceptor modulation of contractile activity of the vascular smooth muscle $(16,17)$, the increased vascular permeability caused by NEM may be in part attributed to functional disturbances of such nonreceptor systems responsible for maintenance of normal vascular integrity. Although NEM did not alter the mucosal $\mathrm{SH}$ levels, endogenous $\mathrm{SH}$ may be funtionally linked to the regulation of the microvascular permeability and thereby is important in protecting the gastric mucosa against damage. It may be possible that adrenalectomy, similar to NEM, influences 
such $\mathrm{SH}$ dependent systems without reducing endogenous SH levels and enhances the mucosal vascular permeability and lesions.

It is intriguing that $d m P G E_{2}$ rather worsened ethanol-induced gastric lesions in rats following adrenalectomy or administration of NEM. We have previously shown that the phenomenon of cytoprotection induced by a variety of drugs including $\mathrm{dmPGE}_{2}$ is accompanied by inhibition of gastric motility and proposed that relaxation of the stomach smooth muscle may be involved in the mechanism of mucosal protection against necrotizing agents (18-21). Inhibition of the stomach contraction would change the necrotizing agents from a "strong irritant" to a "mild irritant" through flattening of the mucosal foldings, and it reduces the severity of macroscopically visible lesions induced by necrotizing agents. However, both adrenalectomy and NEM may induce vascular fragility and sensitize the vasculature to irritants. Under these situations, even though dmPGE 2 by inhibiting the stomach contraction dissoluted the mucosal foldings and reduced the irritative activity of ethanol, it seemed noxious enough to induce hemorrhages in the mucosa. This speculation would be supported by the finding that the morphology of the damage was changed from elongated bands into more generalized lesions in the presence of $d m \mathrm{PGE}_{2}$ (in the absence of mucosal folds).

Taken together, the results of the present study suggested that adrenalectomy worsened the mucosal ulcerogenic responses caused by ethanol and abolished the protective effect of $\mathrm{dmPGE}_{2}$, probably by enhancing the microvascular permeability in the gastric mucosa. These effects may be due to a lack of steroid secretion caused by adrenalectomy, but not related to a mucosal $\mathrm{SH}$ deficiency.

\section{References}

1 Hernandez, D.E., Adcock, J.W., Nemeroff, C.B., Orlando, R.C. and Prange, A.J.: The effect of adrenalectomy and hypophysectomy on neurotensin-induced cytoprotection against stressinduced gastric ulcers in rats. Soc. Neurochem. Sym. 8, 460 (1980)

2 Szabo, S., Gallagher, G.T., Horner, H.C., Frankel, P.W., Underwood, R.H., Konturek, S.J., Brozozowski, T. and Trier, J.S.: Role of the adrenal cortex in gastric mucosal protection by prostaglandins, sulfhydryls, and cimetidine in the rat. Gastroenterology 85, 1384-1390 (1983)

3 Derelanko, M.J. and Long, J.F.: Influence of prednisolone on ethanol-induced gastric injury in the rat. Dig. Dis. Sci. 27, 149-154 (1982)

4 Szabo, S., Trier, J.S., Brown, A. and Schnoor, J.: Early vascular injury and increased vascular permeability in gastric mucosal injury caused by ethanol in the rat. Gastroenterology $88,228-236$ (1985)

5 Pihan, G., Majzoubi, D., Haudenschild, C., Trier, J.S. and Szabo, S.: Early microcirculatory stasis in acute gastric mucosal injury in the rat and prevention by 16,16-dimethyl prostaglandin $E_{2}$ or sodium thiosulfate. Gastroenterology 91, 1415-1426 (1986)

6 Szabo, S., Trier, J.S. and Frankel, P.W.: Sulfhydryl compounds may mediate gastric cytoprotection. Science 214, 200-202 (1981)

7 Takeuchi, K., Furukawa, O., Nishiwaki, H. and Okabe, S.: 16,16-Dimethyl prostaglandin $E_{2}$ aggravates gastric mucosal injury induced by histamine in rats; Possible role of the increased mucosal vascular permeability. Gastroenterology 93, 1276-1286 (1987)

8 Katayama, S., Shionoya, H. and Ohtake, S.: A new method for extraction of extravasated dye in the skin and the influence of fasting stress on passive cutaneous anaphylaxis in guinea pigs and rats. Microbiol. Immunol. 22, 89-101 (1978)

9 Kaplowitz, N., Kuhlenkamp, J., Goldstein, J.L. and Reeve, J.: Effect of salicylates and phenobarbital on hepatic glutathione in the rat. J. Pharmacol. Exp. Ther. 212, 240-245 (1980)

10 Dunnett, C.W.: A multiple comparison procedure for comparing several treatments with a control. J. Am. Stat. Assoc. 50, 1096-1121 (1955)

11 Urushidani, T., Kasuya, Y. and Okabe, S.: The mechanism of aggravation of indomethacininduced gastric ulcers by adrenalectomy in the rat. Japan. J. Pharmacol. 29, 775-780 (1979)

12 Szabo, S. and Szelenyi, I.: Cytoprotection in gastrointestinal pharmacology. Trends Pharmacol. Sci. 8, 149-154 (1987)

13 Moncada, S., Ferreira, S.H. and Vane, J.R.: Prostaglandins as potentiators of increased vascular permeability in inflammation. Nature 246, 215-219 (1973)

14 Oyanagi, Y.: Physiological regulation of vascular permeability by endogenous glucocorticoids and active oxygen. Inflammation 7, 81-89 (1983)

15 Szabo, S. and Trier, J.S.: Pathogenesis of acute gastric mucosal injury: Sulfhydryls as a protector. adrenal cortex as a modulator, and vascular 
endothelium as a target. In Mechanisms of Mucosal Protection in the Upper Gastrointestinal Tract, Edited by Allen, A., Flemstrom, G., Garner, A., Silen, W. and Turnberg, L.A., p. 287-293, Raven Press, New York (1984)

16 Neering, D.R. and Glover, W.E.: The role of sulfhydryl groups in contraction of vascular smooth muscle. J. Pharmacol. Exp. Ther. 208, 335-340 (1979)

17 Ghanayem, B.I., Boor, P.J. and Ahmed, A.E.: Acrylnitrile-induced gastric mucosal necrosis: Roles of gastric glutathione. I. Pharmacol. Exp. Ther. 232, 570-577 (1985)

18 Takeuchi, K. and Nobuhara, Y.: Inhibition of gastric motor activity by 16.16-dimethyl prostaglandin $E_{2}$ : A possible explanation of cyto- protection. Dig. Dis. Sci. 30, 1181-1188 (1985)

19 Takeuchi, K., Nishiwaki, H. and Okabe, S.: Cytoprotective action of mast cell stabilizers against ethanol-induced gastric lesions. Japan. J. Pharmacol. 42, 297-307 (1986)

20 Takeuchi, K., Nishiwaki, H., Ishihara, Y. and Okabe, S.: Roles of gastric motility changes in cytoprotection induced by acetazolamide and cysteamine in rats. Japan. J. Pharmacol. 44, 269-281 (1987)

21 Takeuchi, K., Nishiwaki, H., Okada, M. and Okabe, S.: Mucosal protective action of histamine against mucosal lesions induced by $\mathrm{HCl}$ in rats: Importance of antigastric motor activity mediated by $\mathrm{H}_{2}$-receptors. J. Pharmacol. Exp. Ther. 245, 632-638 (1988) 